\title{
Resonant acoustic waves in a stratified atmosphere
}

\author{
Y. Taroyan and R. Erdélyi \\ $\mathrm{SP}^{2} \mathrm{RC}$, Department of Applied Mathematics, University of Sheffield, Sheffield S3 7RH, UK \\ email: y.taroyan; robertus@sheffield.ac.uk
}

\begin{abstract}
The upward propagation of linear acoustic waves in a gravitationally stratified atmosphere is studied. The wave motion is governed by the Klein-Gordon equation which contains a cut-off frequency introduced by stratification. The acoustic cut-off may act as a potential barrier when the temperature decreases with height. It is shown that waves trapped below the barrier could be subject to a resonance which extends into the entire unbounded atmosphere. The parameter space characterizing the resonance is explored.
\end{abstract}

Keywords. waves, hydrodynamics, Sun: atmosphere, Sun: photosphere, Sun: chromosphere, Sun: Corona, Sun: oscillations

\section{Introduction}

Acoustic waves have often been invoked as possible candidates for the heating of solar and stellar chromospheres and coronae (see e.g. Banerjee et al. 2007 for the latest review on observations; and Erdélyi 2004; 2007 on theory). Until recently it was thought that high frequency waves could be responsible for the heating of the non-magnetic chromosphere. On the other hand, low frequency waves were believed to play little role as far as the dynamics and energetics of the atmosphere are concerned due to reflection from regions with steep temperature gradients. Recent works have changed these views. It was established that the power of the observed high frequency propagating $(>5 \mathrm{mHz})$ acoustic waves is not enough to balance the radiative losses in the chromosphere (Fossum \& Carlsson 2005). On the other hand, new observations have shown that the energy flux carried by the low frequency $(<5 \mathrm{mHz})$ acoustic waves into the chromosphere is about a factor of 4 greater than that carried by high frequency waves (Jefferies et al. 2006). It was argued that these low frequency waves could propagate and carry their energy into the higher layers of the atmosphere through portals formed by the inclined magnetic field lines. Further, it was demonstrated that dynamic features such as solar chromospheric spicules or fibrils could be associated with the leakage of global $p$-modes into the atmosphere along inclined field lines (De Pontieu et al. 2004). A strong correlation was also found between propagating intensity oscillations in the corona and $p$-modes (De Pontieu et al. 2005). These and other results have prompted renewed strong interest in the theory of low frequency acoustic wave propagation in stratified media.

The present paper deals with a two-layer model (Fig. 1) to study the vertical propagation of acoustic waves in a stratified atmosphere (either plasma or gaseous). The waves are described by the Klein-Gordon (KG) equation. The main result here is the discovery of a resonance occurring at low frequencies which extends into the entire unbounded atmosphere. This previously unknown resonance may be responsible for the transfer of wave energy which could have dynamic consequences and heat the higher atmospheric layers. The KG equation is widely used in a range of fields such as atmospheric physics, cosmology, quantum field theory, solid state physics, solar/stellar physics. Therefore, the 
presented results may have wider applicability in distinct areas of physics and astrophysics.

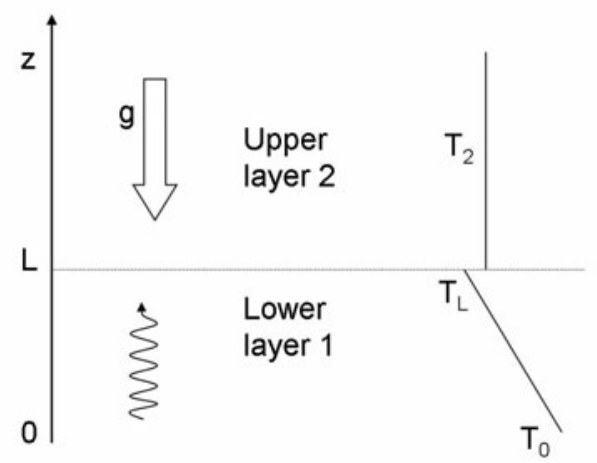

Figure 1. Two-layer model depicting a stratified solar atmosphere. The lower part of the atmosphere (index 1) is separated from the upper part (index 2) by a density and temperature discontinuity at $z=L$. Waves are launched at $z=0$ and propagate in the vertical $z$-direction.

\section{Model and Governing Equations}

The proposed one dimensional model is shown in Fig. 1. The atmosphere is stratified under gravity in the $z$ direction. The temperature $T=T(z)$ linearly decreases in the lower part and remains constant in the upper part of the atmosphere:

$$
T= \begin{cases}T_{0}(1-a z), & 0<z<L, \\ T_{2}, & z>L,\end{cases}
$$

where the constant $a\left(a L=1-T_{L} / T_{0}>0\right)$ characterizes the steepness of temperature decrease from $T_{0}=T(0)$ to $T_{L}=T(L)$, and $L$ is the thickness of the nonuniform layer. The wave motion is governed by the $\mathrm{KG}$ equation:

$$
\frac{\partial^{2} Q}{\partial t^{2}}-c^{2}(z) \frac{\partial^{2} Q}{\partial z^{2}}+\Omega^{2}(z) Q=0
$$

where $Q$ is the scaled velocity (Roberts 2004) and $c=(\gamma R T)^{1 / 2}$ is the sound speed. The quantity $\Omega$ represents the acoustic cut-off frequency, which imposes a time-scale on the system:

$$
\Omega^{2}=\frac{c^{2}}{4 \Lambda^{2}}\left(1+2 \frac{d \Lambda(z)}{d z}\right)
$$

where $\Lambda$ is the pressure scale-height proportional to the temperature. An extensive review on solar applications of the KG equation is presented by Roberts (2004). In the present work, the KG equation (2.2) is applied to the study of waves driven at a boundary of a semi-infinite non-isothermal atmosphere:

$$
\lim _{z \rightarrow 0} Q(z, t)=I(\omega) \cos (\omega t)
$$

where $\omega$ is the driver frequency and $I=I(\omega)$ is the frequency dependent amplitude of the driver. For simplicity, we assume that $Q$ is a complex variable. The boundary 
condition (2.4) is then replaced with

$$
\lim _{z \rightarrow 0} Q(z, t)=I(\omega) e^{-i \omega t}
$$

\section{Results and Discussion}

We seek steady state solutions of the form $Q(z, t) \sim \exp (-i \omega t)$. In the lower part of the atmosphere, Eq. (2.2) can be transformed to a Bessel equation. It possesses solutions of the form

$$
Q(z, t)=\exp (-i \omega t) \sqrt{1-a z}\left[A_{1} J_{\nu}\left(\frac{2 \omega}{c_{0} a} \sqrt{1-a z}\right)+B_{1} Y_{\nu}\left(\frac{2 \omega}{c_{0} a} \sqrt{1-a z}\right)\right],
$$

where $J_{\nu}$ and $Y_{\nu}$ are the Bessel functions of the first and second kind, respectively and $\nu=\gamma g /\left(a c_{0}^{2}\right)-1$ is the polytropic index. In the upper layer $z>L$, the solution has the form

$$
Q(z, t)=A \exp (i k z-i \omega t), \quad \text { where } k= \begin{cases}i \frac{\sqrt{\Omega_{2}^{2}-\omega^{2}}}{c_{2}}, & \omega<\Omega_{2}, \\ \frac{\sqrt{\omega^{2}-\Omega_{2}^{2}}}{c_{2}}, & \omega>\Omega_{2},\end{cases}
$$

with $c_{2}$ and $\Omega_{2}$ being the constant sound speed and cut-off frequency in the upper layer. The coefficient $A$ is uniquely determined by appropriately matching the solutions across $z=L$. It determines the wave amplitude in the region above $z=L$. In the WKB limit the coefficient $A$ is reduced to

$$
A=\frac{I(\omega) \exp (i k L)}{1-\frac{L}{2 \Lambda_{2}}+L \frac{\sqrt{\Omega_{2}^{2}-\omega^{2}}}{c_{2}}}
$$

Eq. (3.3) is valid when the lower layer is thin. It shows that the wave amplitude may become infinite in the adopted linear approximation. A necessary condition for the existence of a resonance is $L / 2 \Lambda_{2}>1$. The waves are resonantly amplified when

$$
\omega=\frac{c_{2}}{L} \sqrt{\frac{L}{\Lambda_{2}}-1}
$$

In Fig. 2, the scaled resonant frequency $\omega$ is plotted against the scaled thickness $L$. The temperature $T_{2}$ is fixed (and so are the cut-off frequency $\Omega_{2}$ and the scale height $\Lambda_{2}$ ). Four different cases with different temperature ratios $\left(T_{0} / T_{2}=2,5,8,15\right)$ are shown. In all four cases, $T_{L}=T_{2}$ is set. The resonant frequencies consecutively appear and decrease as the thickness of the lower layer $L$ increases. In general, the higher the length ratio $L / \Lambda$ the lower the temperature ratio $T_{0} / T_{2}>1$ required for the existence of a resonance.

The physical mechanism responsible for wave amplification is the following: the decreasing temperature results in an increasing acoustic cut-off frequency $\Omega=\Omega(z)$ which forms a potential barrier similar to the one in quantum mechanics (Landau \& Lifshitz 1977). Low frequency waves with $\Omega_{0}<\omega<\Omega_{2}$ driven at $z=0$ are reflected back from the barrier and trapped in the lower layer 1 . When the driver frequency matches the natural frequency of the cavity where the waves are trapped a standing wave is set up and amplified resonantly. In the case of a thin layer, only the fundamental mode is present with a frequency given by Eq. (3.4). The frequency of the fundamental mode decreases and higher harmonics appear as the thickness $L$ increases. The resonance affects the evanescent tail of the waves in the upper atmosphere leading to a global resonance. 


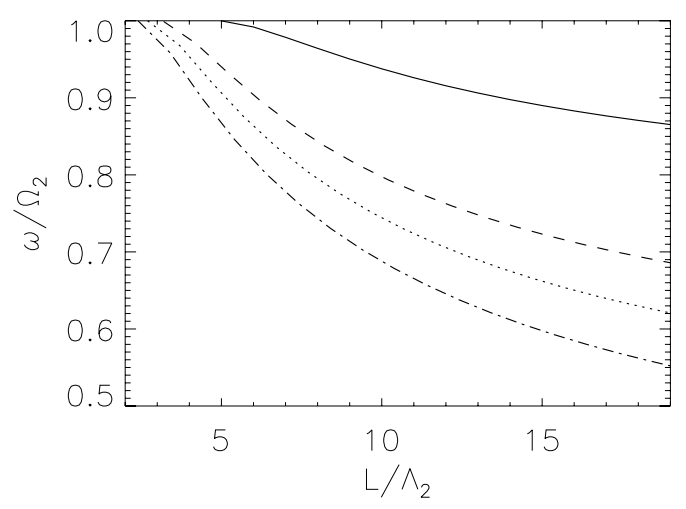

Figure 2. Scaled resonant frequency $\omega$ as a function of the length of the non-uniform layer $L$. Four different cases are shown: $T_{0} / T_{2}=2$ (solid line), $T_{0} / T_{2}=5$ (dashed line), $T_{0} / T_{2}=8$ (dotted line) and $T_{0} / T_{2}=15$ (dash-dotted line). In all four cases, $T_{L}=T_{2}$ is set.

The nonlinear development and dissipation of such waves generated in various physical systems and their energetic implications must be treated separately. These may include the generation of spicules or the heating of the lower atmosphere. An important extension of the present work is the treatment of the problem in $2 \mathrm{D}$ and the consideration of a non-monochromatic driver. The resonant waves presented here arise due to the variation of the cut-off frequency introduced by stratification. However, such waves could operate in any other systems with varying cut-off frequencies. The cut-off frequencies of waves, such as Alfvén, kink and slow waves, in thin magnetic flux tubes vary due to, e.g., crosssection expansion of the flux tube or the variations in the magnetic field strength and density (Spruit \& Roberts 1983). Therefore, such waves could readily become subject to a resonance in solar/stellar structures. The applicability of the presented mechanism in other areas of physics and astrophysics is open for discussion.

\section{Acknowledgements}

Y.T. is grateful to the Leverhulme Trust for financial support. R.E. acknowledges M. Kéray for patient and is also grateful to NSF, Hungary (OTKA, Ref. No. K67746). Both authors thank the IAU for the travel support they have received.

\section{References}

Banerjee, D., Erdélyi, R., Oliver, R. \& O'Shea, E. 2007 Solar Phys., 246, 3

H. Lamb, Hydrodynamics, Cambridge University Press, Cambridge, 1953

Fossum, A. \& Carlsson, M. 2005, Nature, 435, 919

Jefferies, S.M., McIntosh, S.W., Armstrong, J.D. et al. 2006, ApJ, 648, L151

De Pontieu, B., Erdélyi, R., \& James, S.P. 2004 Nature, 430, 546

De Pontieu, D., Erdélyi, R. \& De Moortel, I. 2005, ApJ, 624, L61

Erdélyi, R. 2004, Astron. Geophys., 45, 4.34

Erdélyi, R. 2007 AIP-CP, 919, 122

Roberts, B. 2004, in: R. Erdélyi, J.L. Ballester \& B. Fleck (sci eds.) SOHO 13 Waves, Oscillations and Small-Scale Transients Events in the Solar Atmosphere: Joint View from SOHO and TRACE, ESA-SP, 547, 1

Landau, L.D. \& Lifshitz, E.M. Quantum Mechanics, Pergamon Press, Oxford, 1977

Spruit, H.C. \& Roberts, B. 1983, Nature, 304, 401 